\title{
Comparison the effect of incentive spirometry and conservative therapy among open heart surgery patients for the prevention of postoperative pulmonary complications
}

\author{
Mona AbdElaziem Ahmad, Sanaa Mohammed Ahmad Alaa Eldeen, Hamdy Abbas Youssef \\ \& Mona Ali Mohammed. \\ Assistant lecturer of Critical Care Nursing, Faculty of Nursing, Assiut University Egypt. \\ Professor of Medical \& Surgical Nursing, Faculty of Nursing - Alexandria University Egypt. \\ Professor of Anesthesia \& Intensive care, Faculty of medicine- Assuit University Egypt. \\ Lecturer of Critical Care Nursing, Faculty of Nursing, Assiut University Egypt.
}

\begin{abstract}
patients under Open heart surgery, high risk for many respiratory complications post operatively, chest physiotherapy techniques are used to increase lung volumes, improve oxygenation and decrease the incidence and severity of pulmonary complications after cardiac surgery .deep breathing is a major part of the therapy. Aim ,This study amid to compare the effect of incentive spirometer, and conservative therapy among open heart surgery patients for the prevention of postoperative pulmonary complications. Design, Aquasi -experimental design. Setting, conducted in postoperative intensive care unit of Assiut university hospitals. Subject, a convenience sample of 50 adult open heart patients of both sexes.Group1 (incentive spirometry), group2 (conservative therapy).25 patients for each. Tools, Two tools were developed in this study, tool one (Patient's socio demographic characteristics and health status among open heart patients), tool two (Observation checklist for post-operative pulmonary complications among open heart surgical patients).Result of this study revealed that a statistical significant difference was found between the two groups regarding to atalectasis . As regard pulmonary secretion it was noticed that a highly percent $80 \% \& 72 \%$ of patients in group 2 (conservative therapy) \&1(incentive spirometer) respectively. Conclusion of this study illustrated that a positive effects of incentive spirometer compared with other physiotherapy breathing techniques on occurrence of pulmonary complications among open heart patients postoperatively, showed a significantly smaller amount of secretions and atelectasis, improved oxygenation and had less reduction in FVC(forced vital capacity) and FEV1 (forced expiratory volume in one second)on the fourth postoperative day compared to conservative therapy. Recommendations, Explain to the nurse the deference between deep breathing exercise alone and deep breathing exercise with anther maneuver.
\end{abstract}

Key words: incentive spirometer, conservative therapy, postoperative pulmonary complications \& open heart surgery.

\section{Introduction}

The open-heart surgical patient presents the great challenge to nurses in intensive care units (Hery\& Thompson, 2005). Open-heart surgical patients are exposed to the occurrence of postoperative pulmonary complications due to the effect of cardiopulmonary bypass machine, anesthesia, and compression of the lung tissue from injury to the phrenic never that causes diaphragmatic dysfunction and postoperative incisional pain (Sculler\& Marrow, 2007).

Postoperative pulmonary complications (PPC) present high rates of morbidity, mortality, increased hospital costs and prolonged hospital stay predominantly in abdominal, cardiac and thoracic surgery(Waissman, 2004 \& Smetana, 2009). The incidence of PPC varies according to the previous diagnosis of the candidates for surgery and the type of surgery (Canet et al., 2010). For all these reasons, the incidence rates vary dramatically, ranging from 2 to $40 \%$ Atelectasis, pneumonia, tracheobronchitis, bronchospasm, exacerbation of chronic obstructive pulmonary disease, acute respiratory failure and prolonged mechanical ventilation (longer than 48 hours) can be classified as PPCs (Lawrence et al., 2004 \& Joehl, 2005). The major causes of PPCs may be related to shallow breathing and monotonous tidal volume in post-operative patients (Bartlett et al., 2010), other causes such as anesthesia, opioid analgesia, and postoperative pain also seem to contribute to this ventilation pattern without spontaneous deep breaths that occurs every 5 or 10 minutes (Duggan \& Kavanagh, 2010).Moreover, presence of risk factors as underling cardiac pathology, smoking, chronic obstructive pulmonary disease, age, and obesity increase the incidence of pulmonary complications (Curley et al., 2006) .Comprehensive pulmonary care can prevent a prolonged stay in intensive care unit with its many attendant complications. 
Chest physiotherapy is routinely used in order to prevent or reduce pulmonary complications after surgery, was initially aimed to improve ventilation and oxygenation by removing secretions from the airways. In an effort to increase lung volume following surgery, various deep breathing maneuvers have been implemented as a main component in the care of the postoperative patient. Today, Postoperative treatment includes early mobilization, change position breathing exercises and coughing techniques (Ingwersen et al., 2005). various chest physiotherapy techniques are used after cardiac surgery for example incentive spirometer, continuous positive airway pressure and intermittent positive pressure breathing, positive expiratory pressure (PEP) as blow bottle is a another technique to produce expiratory resistance and initial rationale for the technique was to expand the lungs, (Westerdah et al., 2001 \& Savci et al., 2006). Use of incentive spirometer has been widely reported as an intervention post operatively either as prophylaxis or for treatment of respiratory complications ..

Incentive spirometers, referred to as sustained maximal inspiration devices (SMI), an incentive spirometer is a handheld device that exercises the lungs and measures how much air can breathe and tells about how the lungs are working (Deborah Leader, 2008).

A flexible plastic tube is connected to a large and small air column. The large column has a piston or ball that moves up each time you breathe in. This column measures how much air you breathe in the effort is marked in units of milliliters (Thompson et al., 2010).

Purpose of incentive spirometer, measure the flow of air inhaled through the mouthpiece and are used to improve pulmonary ventilation, after surgery especially thoracic and abdominal surgery, counteract respiratory secretions, facilitate respiratory gaseous exchange, and prevent or treat atelectasis, loosen respiratory secretion and expand collapsed alveoli. Incentive spirometry is designed to mimic natural sighing by encouraging the client to take long, slow, deep breathing, Open the airway and make it easier to breathe, Prevent a buildup of fluid and mucus in lungs, Prevent serious lung infections like pneumonia, Manage the symptoms of lung disease such as COPD and lungs active if you're on bed rest. (Weindler \& Kiefer, 2011).

Several rehabilitation strategies have been applied to these patients in order to minimize the alterations of respiratory function. Although there is no consensusin the literature about the best physiotherapy respiratory intervention in patients undergoing CABG surgery, it seems that the application of noninvasive positive pressure ventilation improves lung function compared to conventional respiratory therapy (Pasquina \& Martin, 2005).

Critical care nurse plays a critical role in returning open-heart surgical patient to optimal level of functioning, promoting healing and preventing complications during assessment, the nurse gat her information that is important to determine respiratory status and plan patient's care. The nurse assesses breath sounds, respiratory rate and depth, oxygen saturation, arterial blood gases results, pulmonary function studies, and diagnostic chest X-ray films. These results are valuable in predicting postoperative respiratory function and reducing incidence of postoperative pulmonary complications. And, the nurse should assess patient's tolerance during treatment (Black \& Jacobs, 2010).

\section{Aim of the Study}

This study aimed to

- Compare the effect of incentive spirometry, and conservative therapy on the prevention of postoperative pulmonary complications after open heart surgery patients.

Research hypotheses

To fulfill the aim of the study following research hypothesis were formulated:

- Post-operative open heart surgery patients practicing routine deep breathing and coughing exercise have expressing sever incidence of pulmonary secretion than other maneuvers

- Post-operative open heart surgery patients using incentive spirometry expressing moderate pulmonary secretion and improve expansion of the lung than other maneuvers

\section{Subject and Method \\ Research Design:}

A quasi -experimental design was utilized to fulfill the aim of this study .This design was used to explain relationships, clarify certain events happened or both.

\section{Material}

Variables

- Independent variables in this study are deep breathing and coughing exercises (conservative therapy), and incentive spirometer.

- Dependent variables are patient's respiratory complications.

\section{Setting}

The study was conducted in postoperative intensive care unit at Assiut university hospitals.

\section{Subject}

A convenience sample of 50 adult open heart patients of both sexes constitutes the study sample. The subjects were assigned into two groups. Group1 
(incentive spirometry), and group2 (conservative therapy), 25 patients for each.

Inclusions criteria

- adult aged from 20-50 years,

- Extubated within 4-6 hours after open heart surgery,

- End expiratory pressure $<5 \%$,

- No fever in first four days postopratively,

- No respiratory disease,

- Fraction inspired oxygen $>40 \%$ on mechanical ventilator and No pain after operation.

\section{Study Tools}

Two tools were developed and used by the researcher in this study after reviewing the related literatures (westerdhi, 2004).

\section{Tool I}

patient's socio demographic characteristics and health status assessment sheet among open heart surgery patients, this tool was includes six parts .

\section{Part I}

Assessment of the patient personal data as patient name, age, sex, marital status, occupation .....)

\section{Part II}

\section{clinical medical data which include:}

past medical history for(Cyanosis, Streptococcal infections, Rheumatic fever, Diseases as heart failure, Allergies ), medical diagnose, date of admission, date of intubation on mechanical ventilion, patient weight and height, Times from extubation, Duration of operation, Type of operation, Data of operation, ,Time of operation, time of chest tube removal.

\section{Part III}

- Preoperative medications (Intropics, Coronary vasodilator, Anti-hypertension, diuretics, hypoglycemic, others).

- Health habits (Use of tea and coffee, Use of alcohol, Smoking, Exercise) .

- Hereditary diseases (Diabetes, Renal disease, congenital heart disease and hypertension) and previous cardiac surgery.

Part VI

- Homodynamic parameters (vital signs): temperature and respiratory rate.

\section{Part V}

Post operative open heart surgical patient assessment before and after extubation, consist of:

A - Assessment of Respiratory system before extubation includes:

- Assessment of the initial ventilator parameter at the time of study:

As: - Fio2 (fraction inspired oxygen)

- $\quad$ PEEP (positive end expiratory pressures)

- $\quad$ Ps (pressure support)

- $\quad$ Mode (SIM, Ps, spontaneous)
- Frequency ( rate of inspiration )

- Vt ( tidal Volume)

- Inspiratory to expiratory ratio(I:E)

B-Assessment of Respiratory system after extubation includes: Chest condition as respiratory rate, Depth, Cough (Dry or productive cough), Sputum characteristics: amount, viscosity and color, Dyspnea, Orthopnea 'chest pain,pain with breathing and Auscultation findings to assess of breathing sound as (crepitating, wheezing...).

C-Postoperative numerical sternotomy incision pain scale includes (Pain at rest, Pain while taking deep breath, Pain while coughing, Pain at pulmonary function test)

Scoring: non (0) mild (1-3)

moderate (4-6) sever (7-10)

D-laboratory investigation includes: $\mathrm{CBC}$ (complete blood count) before operation and in third day after operation (white blood cells)

\section{Part IV}

This part includes evaluation of respiratory system by using:

- ABG (Arterial Blood Gas), Six times every 4 hours in the first and second day postoperatively after extubation and three times every 8 hours in third and fourth day postoperatively, take mean of each day.

- Pulmonary function test done (preoperative and in fourth day postoperative open heart patient).Different measurements that may be found on your report after spirometry include: Forced vital capacity (FVC), Forced expiratory volume on one second (FEV1), vital capacity (VC)

- Chest X-ray was done preoperative and in fourth day postoperative open heart surgery.

Tool two

evaluation tool for post-operative pulmonary complications among open heart surgery patients: (Appendix II)

Comparison the effect of (conservative therapy, and incentive spirometer) on occurrence of postoperative pulmonary complications.

Includes mission:

- Atelectasis, (through auscultation ,chest x-ray, pulmonary function test, and blood gas)

- Pneumonia(through auscultation ,chest x-ray, and blood gas)

- Bronchitis(through auscultation ,chest x-ray, and blood gas)

- fever(through oral temperature)

- cough with blood or sputum.

Methods

The study was conducted throughout three main 
phases, which are preparatory phase, implementation and evaluation phase

\section{Preparatory phase}

- An official approval and administration permission were obtained from the head of internal cardiothoracic surgery department and postoperative ICU to collect the necessary data, the aim of the study and the program was explained to them to obtain their cooperation.

- The study was approved by the local ethical committee at faculty of nursing at Assiut University.

- Protection of human rights (Ethical consideration): Informed consent was done obtained from each patient after explain the aim of the study with confidentiality of data for research purpose.

- Content validity: The tools were tested for content validity of research by Jury of 5 expertise from the field of staff thoracic surgery (2 professors and 1 assistant professor) and nursing educators (2 professors).

- Pilot study: A pilot study was conducted on 5 patients to test feasibility and applicability of the tools, the analysis of the pilot study revealed that minimal modifications are required. These necessary modifications were donning and the subjects were excluded from the actual study.

- The data collection covered a period of one year and 9 months starting from September 2011 till the end of June 2013.

- The researcher was interviewing the patients individually to collect the necessary data.

Implementation and evaluation phase

- Group1 was received chest physiotherapy (by incentive spirometer) for 5 to 10 times per day every 1 to 2 hours in second, third and fourth postoperative days, patient inhales slowly and deeply through the mouth into incentive spirometer, holding breath for 2 to 3 second, observe the patient progress by watching the ball elevate, after that exhale around mouthpiece and breath normally for several breath, then after this maneuver patient done deep and coughing exercise for removing secretion. Ask the patient to repeat this procedure along the day.

- Group2 was received usual routine chest physiotherapy (deep and coughing exercise, precaution, and vibration) for patients with open heart surgery (conservative therapy).

For two groups

- All patients in two groups receive analgesic before done any procedure.

- Assess gas exchange by arterial blood gas ABG (arterial blood gas), 6 times every 4/24 hour in the first two days (before extubation, after extubation and Three times every $8 / 24$ hour in the third and fourth day postoperatively after extubation and after done deep breathing maneuvers within 30 muint, then take mean of each day.

- Assess vital signs every two hours in:

- preoperative day (1st assessment) and four assessment postoperatively in first day

- first post-operative day(2nd assessment)

- second post-operative day(3thassessment)

- third post-operative day (4th assessment)

- And fourth post -operative day (5th assessment), then take mean of each day.

- A comparison was done between the two groups to assess occurrence of respiratory complications in fourth day (Appendix II).

- A comparison was done between the two groups for pulmonary function test, done in preoperative day (1st assessment) and in fourth postoperative day (2nd assessment). Different measurements that may be found on your report after spirometry includes: Forced vital capacity (FVC), Forced expiratory volume on one second (FEV1), vital capacity (VC).

- Assess Postoperative sternotomy incision pain, pain measured by numerical scale: include (Pain at rest, Pain while taking deep breath, Pain while coughing, Pain at pulmonary function test)

Evaluation phase

- Evaluate Post-operative pulmonary complications among open heart surgical patients for four groups:

- Atelectasis, (through auscultation ,chest x-ray, pulmonary function test, and blood gas)

- Pneumonia(through auscultation, chest x-ray, and blood gas)

- Bronchitis(through auscultation ,chest x-ray, and blood gas)

- fever(through oral temperature)

- cough with sputum or blood

- Observation checklist for each maneuver (appendix 3 ,) which the researcher instruct the patient to done the steps of procedure.

\section{Analysis of data}

Data collected by computer programs through SPSS" version.17" Chicago. USA. Data expressed as "mean \pm standard deviation" "number, percentage". Using T.test to determine significant for numeric variable.Using Chi.squire test to determine significant for non-parametric variable. Using person's correlation for numeric variable in the same group, n.s $\mathrm{P}>0.05$ no significant, $\mathrm{P}<0.05$ significant, $* * \mathrm{P}<0.01$ moderate significan, $* * * \mathrm{P}<$ 0.001 highly significant. 


\section{Results}

Table (1): Distribution of the Scio demographic characteristics among the two groups.

\begin{tabular}{|c|c|c|c|c|c|}
\hline \multirow[t]{2}{*}{ Variable } & \multicolumn{2}{|c|}{$\begin{array}{c}\text { study } \\
(n=25)\end{array}$} & \multicolumn{2}{|c|}{$\begin{array}{l}\text { control } \\
(n=25)\end{array}$} & \multirow[t]{2}{*}{ P-value } \\
\hline & No. & $\%$ & No. & $\%$ & \\
\hline \multicolumn{5}{|l|}{ Age } & \multirow{5}{*}{0.083} \\
\hline 18 - 29 years & 8 & 32.0 & 12 & 48.0 & \\
\hline $30-39$ years & 14 & 56.0 & 6 & 24.0 & \\
\hline $40-49$ years & 2 & 8.0 & 2 & 8.0 & \\
\hline$\geq 50$ years & 1 & 4.0 & 5 & 20.0 & \\
\hline \multicolumn{5}{|l|}{ Sex } & \multirow{3}{*}{--} \\
\hline Male & 17 & 68.0 & 17 & 68.0 & \\
\hline Female & 8 & 32.0 & 8 & 32.0 & \\
\hline
\end{tabular}

Table (2): Distribution of the sample according to preoperative medical history health habits and body measurement for two groups.

\begin{tabular}{|c|c|c|c|c|c|}
\hline \multirow{2}{*}{ Variable } & \multicolumn{2}{|c|}{ Study $(n=25)$} & \multicolumn{2}{|c|}{ Control $(n=25)$} & \multirow{2}{*}{ P-value } \\
\hline & No. & $\%$ & No. & $\%$ & \\
\hline \multicolumn{6}{|l|}{ Health habits } \\
\hline Use tea \& coffee & 13 & 52.0 & 15 & 60.0 & 0.883 \\
\hline Use alcohol & 1 & 4.0 & 5 & .0 & 0.346 \\
\hline Smoking & 11 & 41.0 & 5 & 16.0 & 0.059 \\
\hline \multicolumn{6}{|l|}{ Medical history } \\
\hline Cyanosis & 16 & 64.0 & 7 & 28.0 & $0.011^{*}$ \\
\hline Streptococcal infection & 20 & 80.0 & 17 & 68.0 & 0.333 \\
\hline Rheumatic fever & 17 & 68.0 & 18 & 72.0 & 0.758 \\
\hline heart failure & 3 & 12.0 & 2 & 8.0 & 0.637 \\
\hline Allergies & 0 & 0.0 & 2 & 8.0 & 0.470 \\
\hline dental infection & 7 & 28.0 & 2 & 8.0 & 0.141 \\
\hline Body measurements & \multicolumn{2}{|c|}{ Mean \pm SD } & \multicolumn{2}{|c|}{ Mean \pm SD } & \\
\hline Weight & \multicolumn{2}{|c|}{$60.12 \pm 5.67$} & \multicolumn{2}{|c|}{$62.32 \pm 13.71$} & 0.462 \\
\hline Length & \multicolumn{2}{|c|}{$160.56 \pm 7.60$} & \multicolumn{2}{|c|}{$163.76 \pm 7.51$} & 0.141 \\
\hline BMI & \multicolumn{2}{|c|}{$23.38 \pm 2.38$} & \multicolumn{2}{|c|}{$23.28 \pm 5.13$} & 0.891 \\
\hline
\end{tabular}

Group 1; incentive spirometer method Group 2; conservative method BMI(body mass index)

Table (3): Comparison between two groups among patient stay in ICU and Time for chest tube removal.

\begin{tabular}{|c|c|c|c|}
\hline Items & Study $(n=25)$ & Control $(n=25)$ & P-value \\
\hline \multicolumn{3}{|l|}{ ICU stay } & \multirow{3}{*}{0.382} \\
\hline Mean \pm SD & $4.12 \pm 0.60$ & $4.36 \pm 1.22$ & \\
\hline Range & $3-5$ & $3-8$ & \\
\hline \multicolumn{3}{|c|}{ Time for chest tube removal } & \multirow{3}{*}{0.712} \\
\hline Mean \pm SD & $3.84 \pm 0.75$ & $3.76 \pm 0.78$ & \\
\hline Range & $3-6$ & $3-5$ & \\
\hline
\end{tabular}

Group 1; incentive spirometer method Group 2; conservative method 
Table (4): Comparison between the two studied groups in relation to vital signs during the fifth assessments (preoperative one assessment $\left(1^{\text {st }}\right)$ and fourth assessments postoperatively.

\begin{tabular}{|c|c|c|c|c|}
\hline \multirow{2}{*}{\multicolumn{2}{|c|}{ Vital signs }} & \multirow{2}{*}{$\begin{array}{c}\text { Study }(n=25) \\
\text { Mean } \pm \text { SD }\end{array}$} & \multirow{2}{*}{$\begin{array}{c}\text { Control }(\mathrm{n}=25) \\
\text { Mean } \pm \text { SD }\end{array}$} & \multirow{2}{*}{ P-value } \\
\hline & & & & \\
\hline \multirow{5}{*}{ Respiratory rate } & Pre-operative( $\left(1^{\text {st }}\right)$ & $17.80 \pm 1.91$ & $19.20 \pm 2.06$ & $0.016^{*}$ \\
\hline & $2^{\text {nd }}$ assessment & $20.04 \pm 3.16$ & $19.92 \pm 1.78$ & 0.869 \\
\hline & 3rd assessment & $19.92 \pm 3.16$ & $20.08 \pm 1.53$ & 0.821 \\
\hline & 4th assessment & $20.24 \pm 1.56$ & $20.80 \pm 2.25$ & 0.312 \\
\hline & $5^{\text {th }}$ assessment & $19.56 \pm 2.14$ & $19.96 \pm 2.72$ & 0.566 \\
\hline \multirow{5}{*}{ Temperature } & $\begin{array}{l}\text { Pre-operative }\left(1^{\text {st }}\right) \\
\end{array}$ & $37.13 \pm 0.21$ & $36.71 \pm 0.46$ & $0.000^{*}$ \\
\hline & $2^{\text {nd }}$ assessment & $36.93 \pm 0.28$ & $37.02 \pm 0.40$ & 0.350 \\
\hline & 3rd assessment & $37.42 \pm 0.17$ & $37.28 \pm 0.28$ & $0.036^{*}$ \\
\hline & 4th assessment & $37.29 \pm 0.37$ & $37.45 \pm 0.37$ & 0.129 \\
\hline & $5^{\text {th }}$ assessment & $37.46 \pm 0.36$ & $37.60 \pm 0.52$ & 0.272 \\
\hline
\end{tabular}

Table (5): comparison between two groups in mechanical ventilation (MV) data before extubation postoperatively.

\begin{tabular}{|c|c|c|c|c|c|c|}
\hline \multirow{2}{*}{\multicolumn{2}{|c|}{ Item }} & \multicolumn{2}{|c|}{ Study $(n=25)$} & \multicolumn{2}{|c|}{ Control $(n=25)$} & \multirow{2}{*}{$P$-value } \\
\hline & & No. & $\%$ & No. & $\%$ & \\
\hline \multirow{2}{*}{ Mode of MV } & SIMV & 6 & 24.0 & 6 & 24.0 & \multirow[b]{2}{*}{--} \\
\hline & SPONT & 19 & 76.0 & 19 & 76.0 & \\
\hline & & \multicolumn{2}{|c|}{ Mean \pm SD } & \multicolumn{2}{|c|}{ Mean \pm SD } & \\
\hline \multicolumn{2}{|l|}{ Fio2 } & \multicolumn{2}{|c|}{$38.60 \pm 5.50$} & \multicolumn{2}{|c|}{$38.80 \pm 5.64$} & 0.900 \\
\hline \multicolumn{2}{|l|}{ PS } & \multicolumn{2}{|c|}{$11.96 \pm 2.53$} & \multicolumn{2}{|c|}{$11.64 \pm 2.12$} & 0.630 \\
\hline \multicolumn{2}{|l|}{ PEEP } & \multicolumn{2}{|c|}{$4.76 \pm 0.66$} & \multicolumn{2}{|c|}{$5.00 \pm 0.00$} & 0.077 \\
\hline \multicolumn{2}{|l|}{$\mathrm{F}$ (frequency) } & \multicolumn{2}{|c|}{$12.44 \pm 1.39$} & \multicolumn{2}{|c|}{$13.20 \pm 1.61$} & 0.080 \\
\hline
\end{tabular}

Group 1; incentive spirometer method Group 2; conservative method Fio2 (oxygen concentration)

PS (pressure support) PEEP (positive end expiatory pressure)

Table (6): assessment of respiratory system after extubation.

\begin{tabular}{|c|c|c|c|c|c|}
\hline \multirow[b]{2}{*}{ Items } & \multicolumn{2}{|c|}{ Study $(n=25)$} & \multicolumn{2}{|c|}{ Control $(n=25)$} & \multirow{2}{*}{ P-value } \\
\hline & No. & $\%$ & No. & $\%$ & \\
\hline \multicolumn{5}{|l|}{ Rate } & \multirow{3}{*}{0.552} \\
\hline Normal & 24 & 96.0 & 23 & 92.0 & \\
\hline Tachy apnea & 1 & 4.0 & 2 & 8.0 & \\
\hline \multicolumn{5}{|l|}{ Depth } & \multirow{3}{*}{0.312} \\
\hline Deep & 24 & 96.0 & 25 & 100.0 & \\
\hline Shallow & 1 & 4.0 & 0 & 0.0 & \\
\hline \multicolumn{5}{|l|}{ Cough } & \multirow{3}{*}{$0.010^{*}$} \\
\hline Yes & 6 & 24.0 & 15 & 60.0 & \\
\hline No & 19 & 76.0 & 10 & 40.0 & \\
\hline \multicolumn{5}{|l|}{ Color of secretion } & \multirow{3}{*}{0.103} \\
\hline White & 19 & 76.0 & 24 & 96.0 & \\
\hline Green & 6 & 24.0 & 1 & 4.0 & \\
\hline \multicolumn{5}{|l|}{ Amount of secretion } & \multirow{3}{*}{0.059} \\
\hline Mild & 21 & 84.0 & 15 & 60.0 & \\
\hline Moderate & 4 & 16.0 & 10 & 40.0 & \\
\hline
\end{tabular}

Independent samples $t$-test $\quad *$ Statistical significant difference $(P<0.05)$

Group 1; incentive spirometer method Group 2; conservative method 
Table (7): distribution of sample according to Postoperative pain using numerical scale.

\begin{tabular}{|c|c|c|c|c|c|}
\hline \multirow{2}{*}{ Pain } & \multicolumn{2}{|c|}{ Study $(n=25)$} & \multicolumn{2}{|c|}{ Control $(n=25)$} & \multirow{2}{*}{ P-value } \\
\hline & No. & $\%$ & No. & $\%$ & \\
\hline \multicolumn{5}{|l|}{ Pain at rest } & \multirow{3}{*}{0.508} \\
\hline None(0) & 20 & 80.0 & 18 & 72.0 & \\
\hline Mild(1-3) & 5 & 20.0 & 7 & 28.0 & \\
\hline \multicolumn{5}{|c|}{ Pain while taking deep breath } & \multirow{3}{*}{0.470} \\
\hline Mild(1-3) & 25 & 100.0 & 23 & 92.0 & \\
\hline Moderate(4-6) & 0 & 0.0 & 2 & 8.0 & \\
\hline \multicolumn{5}{|l|}{ Pain while coughing } & \multirow{3}{*}{$0.037 *$} \\
\hline Mild(1-3) & 20 & 80.0 & 13 & 52.0 & \\
\hline Moderate(4-6) & 5 & 20.0 & 12 & 48.0 & \\
\hline \multicolumn{5}{|c|}{ Pain when done pulmonary function test } & \multirow{3}{*}{$0.037 *$} \\
\hline Mild(1-3) & 13 & 52.0 & 20 & 80.0 & \\
\hline Moderate(4-6) & 12 & 48.0 & 5 & 20.0 & \\
\hline
\end{tabular}

Group 1; incentive spirometer method

Group 2: conservative method

Table (8): Comparison between the two studied groups in relation to Laboratory investigations.

\begin{tabular}{|c|c|c|c|c|}
\hline \multirow{2}{*}{\multicolumn{2}{|c|}{ Items }} & $\begin{array}{c}\text { study } \\
(n=25)\end{array}$ & $\begin{array}{l}\text { control } \\
(n=25)\end{array}$ & \multirow[t]{2}{*}{ P-value } \\
\hline & & Mean \pm SD & Mean \pm SD & \\
\hline \multirow{3}{*}{$\begin{array}{l}\text { White blood } \\
\text { cells (WBCs) }\end{array}$} & Pre-operative ( $1^{\text {st }}$ assessment) & $6.30 \pm 1.34$ & $6.22 \pm 2.00$ & 0.867 \\
\hline & $1^{\text {st }}$ day ( $2^{\text {nd }}$ assessment $)$ & $8.40 \pm 2.54$ & $6.55 \pm 1.62$ & $0.003 *$ \\
\hline & 4th day ( $3^{\text {rd }}$ assessment) & $10.14 \pm 2.24$ & $12.98 \pm 4.50$ & 0.732 \\
\hline
\end{tabular}

Table (9): Comparison between the two studied groups in relation to arterial blood gas (ABG) during the mean fourth assessment $\left(1^{\text {st }}, 2 \mathrm{nd}, 3\right.$ rdand the $4^{\text {th }}$ day post operatively).

\begin{tabular}{|c|c|c|c|}
\hline \multirow[t]{2}{*}{ Arterial blood gases } & $\begin{array}{c}\text { study } \\
(n=25)\end{array}$ & $\begin{array}{l}\text { control } \\
(n=25)\end{array}$ & \multirow[t]{2}{*}{ P-value } \\
\hline & Mean \pm SD & Mean \pm SD & \\
\hline \multicolumn{4}{|l|}{ PH } \\
\hline 1st day( 1st assessment) & $7.36 \pm 0.09$ & $7.42 \pm 0.13$ & 0.074 \\
\hline 2nd day (2nd assessment) & $7.39 \pm 0.06$ & $7.46 \pm 0.18$ & 0.073 \\
\hline 3rd day (3rd assessment) & $7.42 \pm 0.05$ & $7.38 \pm 0.05$ & $0.017 *$ \\
\hline 4th day (4th assessment) & $7.39 \pm 0.08$ & $7.41 \pm 0.12$ & 0.537 \\
\hline \multicolumn{4}{|l|}{$\mathrm{PaCO} 2$} \\
\hline 1st day( 1st assessment) & $33.81 \pm 7.90$ & $41.37 \pm 6.71$ & $0.001 *$ \\
\hline 2nd day (2nd assessment) & $38.54 \pm 7.00$ & $35.13 \pm 5.11$ & 0.055 \\
\hline 3rd day (3rd assessment) & $36.04 \pm 7.13$ & $37.86 \pm 6.10$ & 0.337 \\
\hline 4th day (4th assessment) & $38.08 \pm 6.28$ & $39.34 \pm 5.76$ & 0.463 \\
\hline \multicolumn{4}{|l|}{$\mathrm{HCO3}$} \\
\hline 1st day( 1st assessment) & $20.16 \pm 5.33$ & $23.96 \pm 3.42$ & $0.004 *$ \\
\hline 2nd day (2nd assessment) & $23.09 \pm 3.35$ & $21.62 \pm 3.39$ & 0.129 \\
\hline 3rd day (3rd assessment) & $24.41 \pm 3.67$ & $21.83 \pm 4.11$ & $0.023 *$ \\
\hline 4th day (4th assessment) & $23.78 \pm 3.16$ & $21.84 \pm 4.88$ & 0.103 \\
\hline \multicolumn{4}{|l|}{$\mathrm{SaO2}$} \\
\hline 1st day( 1st assessment) & $99.54 \pm 0.45$ & $99.21 \pm 0.49$ & $0.016 *$ \\
\hline 2nd day (2nd assessment) & $98.84 \pm 2.10$ & $99.02 \pm 0.61$ & 0.682 \\
\hline 3rd day (3rd assessment) & $99.29 \pm 0.38$ & $98.42 \pm 1.59$ & $0.010^{*}$ \\
\hline 4th day (4th assessment) & $98.56 \pm 0.96$ & $98.62 \pm 1.43$ & 0.863 \\
\hline
\end{tabular}




\begin{tabular}{|c|c|c|c|}
\hline \multirow[t]{2}{*}{ Arterial blood gases } & $\begin{array}{c}\text { study } \\
(\mathbf{n}=\mathbf{2 5})\end{array}$ & $\begin{array}{l}\text { control } \\
(\mathbf{n}=25)\end{array}$ & \multirow[t]{2}{*}{ P-value } \\
\hline & Mean \pm SD & Mean \pm SD & \\
\hline \multicolumn{4}{|l|}{$\mathrm{PaO} 2$} \\
\hline 1st day( 1st assessment) & $193.36 \pm 51.21$ & $187.92 \pm 42.46$ & 0.684 \\
\hline 2nd day (2nd assessment) & $160.76 \pm 27.26$ & $166.08 \pm 25.40$ & 0.479 \\
\hline 3rd day (3rd assessment) & $146.60 \pm 22.39$ & $147.28 \pm 31.91$ & 0.931 \\
\hline 4th day (4th assessment) & $110.84 \pm 13.83$ & $138.16 \pm 32.95$ & $0.0001^{*}$ \\
\hline
\end{tabular}

Group 1; incentive spirometer method Group 2; conservative method

FEV1: forced expiratory volume in one second

FVC: forced vital capacity

VC: vital capacity

Table (10): Comparison between the two studied groups in relation to complications of respiratory system in fourth day.

\begin{tabular}{|l|c|c|c|c|c|}
\hline \multirow{2}{*}{ Complications } & \multicolumn{2}{|c|}{ Study $(\mathbf{n = 2 5})$} & \multicolumn{2}{c|}{ Control (n= 25) } & \multirow{2}{*}{ P-value } \\
\cline { 2 - 5 } & No. & \% & No. & \% & \\
\hline Pneumonia & 1 & 4.0 & 2 & 8.0 & 0.552 \\
\hline Atalectasis & 0 & 0.0 & 3 & 12.0 & 0.234 \\
\hline Bronchitis & 3 & 12.0 & 2 & 8.0 & 0.637 \\
\hline Fever & 2 & 8.0 & 6 & 24.0 & 0.247 \\
\hline Pulmonary Secretion & 18 & 72.0 & 20 & 80.0 & 0.508 \\
\hline
\end{tabular}

Group 1; incentive spirometer method

Group 2; conservative method

Figure(1): Comparison between the two studied groups in relation to pulmonary function test in preoperative day (1st assessment) and fourth day $\left(2^{\text {nd }}\right.$ assessment $)$ postoperatively.

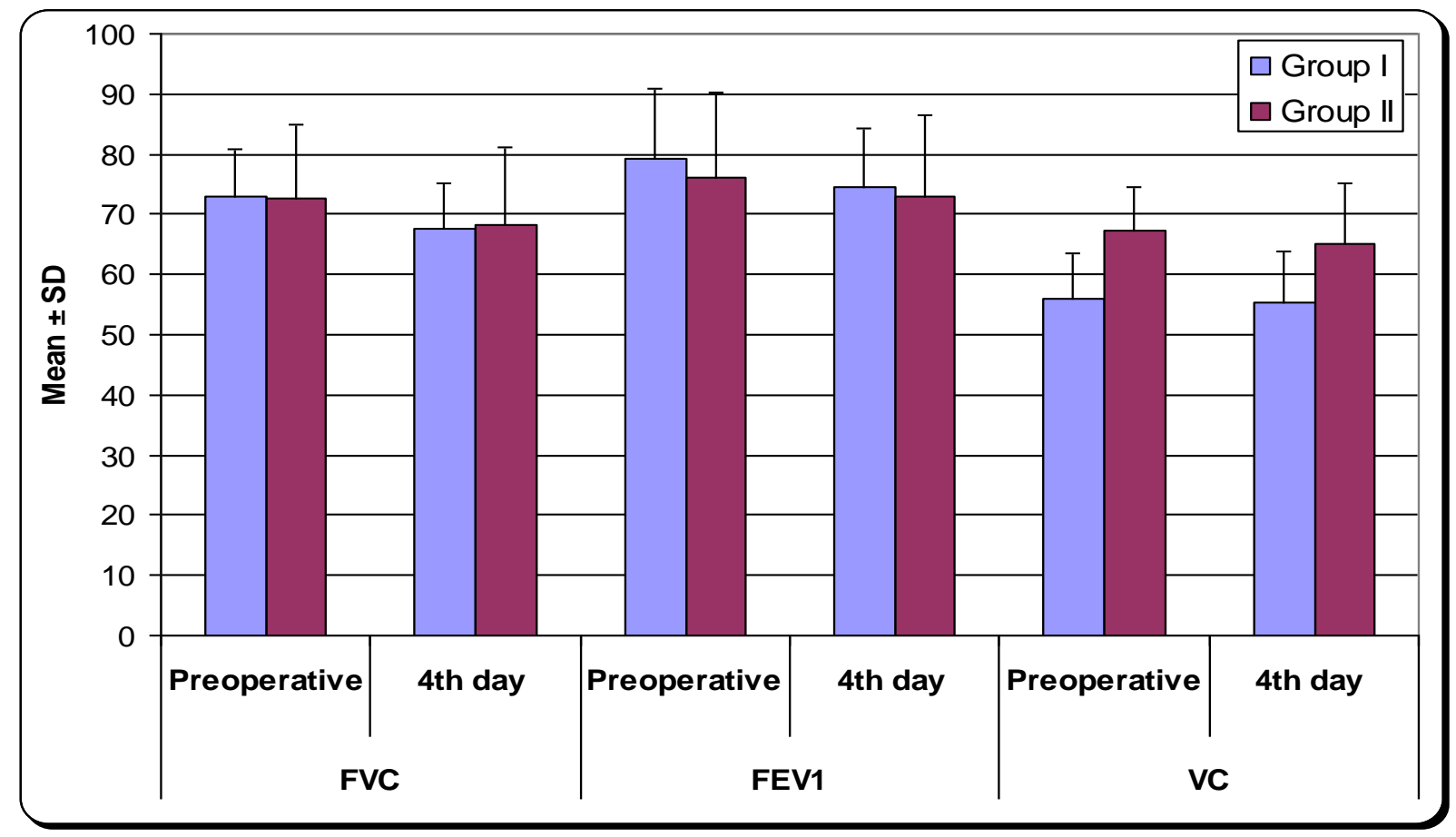

FVC: forced vital capacity

FEV1: forced expiratory volume in one second $\boldsymbol{V C}$ : vital capacity 
Table (1) : Scio demographic characteristics among the two groups, This table demonstrates that $56 \%$ and $56 \%$ of group 1 were in age group 30-39 years old respectively, and $48 \%$ of group 2 was in age group 18 -29 years old with no significant difference between the two groups as regard age. Regard to sex ,it was found that $68 \%$ of group $1 \& 2$ were male with nonsignificant difference between the two groups.

Table (2) : show preoperative medical history health habits and body measurement for three groups. Regarding to health habits it was noticed that $52 \%$ and $60 \%$ of group $1 \& 2$ were used tea\& coffee. As regards smoking .while $41.0 \%$ of patients in groups 1 having smoking with no significant difference between two groups. As regard Medical history the majority of group $1(80 \%)$ were having streptococcal infection and the majority of group $2(72 \%)$ were having rheumatic fever .but ,there were significant difference between two groups as regard Cyanosis $\mathrm{p}(0.011)$. A significant difference between two groups Regarding to body mass index.

Table (3) : patient stay in ICU and Time for chest tube removal. this table show that the mean value of time in ICU stay in group II was $(4.36 \pm 1.22)$ days with non-significance difference between two groups $\mathbf{p}(0.385)$.Regarding to time for chest tube removal ,There was non- significant difference between two groups $\mathbf{p}(0.712)$.

Table (4) : Comparison between the two studied groups in relation to vital signs during the fifth assessments (preoperative one assessment $\left(1^{\text {st }}\right)$ and fourth assessments postoperatively. This table shows that assessment of vital signs in the two groups in preoperative phase and postoperative phase. Concerning the respiratory rate significant differences were found between the two groups during the first assessments with p-value $(0.016)$. Regarding to body temperature, a significant difference was found between the two groups during the first, third, $(\mathrm{p}=0.0001 \& 0.036)$ respectively.

Table (5): mechanical ventilation (MV) data before extubation postoperatively.

This table illustrated that no significant difference was found between the two groups regarding mechanical ventilation (MV) data

Table (6) : assessment of respiratory system after extubation, regarding to assessment of respiratory system after extubation (Cough) this result revealed that a significant difference were found between the two groups $\mathrm{P}(0.010)$.

Table (7) : Postoperative pain using numerical scale, regarding to postoperative pain, it was noticed that $(80 \% \& 52 \%)$ of patients in group $1 \& 2$ respectively having mild pain, while coughing, while $80 \%$ of patients in group 2 having mild pain when done pulmonary function test with statistical significant difference was found between the two groups $\mathrm{P}(0.037)$.As regard pain while taking deep breath it was found $(100 \%, \& 92 \%)$ of patients in group $1 \& 2$ respectively having mild pain while taking deep breath with non-significant difference was found between the two groups.

Table (8) : This table shows that Laboratory investigations in the two groups in preoperative phase and postoperative phase. Concerning the WBCs significant differences were found between the two groups during the second assessments with p-value (0.003).

Table (9) : arterial blood gas (ABG) during the mean fourth assessment $\left(1^{\text {st }}, 2\right.$ nd, 3 rdand the $4{ }^{\text {th }}$ day post operatively), Regarding to $\mathrm{PH}$ it was noticed that a significant difference was found between the two groups during the third assessment $(\mathrm{p}=0.017)$. Concerning the paco2\&HCO3statistical significant differences were found between the two groups during the first assessments with p-value (0.001). In relation to $\mathrm{saO}_{2}$ it was found that significant difference between the two groups during the first and third assessment $(p=0.016 \& 0.010)$ respectively, as regards $\mathrm{paO}_{2}$ a significant difference between the two groups during the fourth assessment $(\mathrm{p}=0.001)$.

Table (10) : complications of respiratory system in fourth day, this table shows that $12 \%$ patients in group 2 having atalectasis with no significant difference was found between the two groups $\mathrm{p}$ (0.234). As regard pulmonary secretion it was noticed that a highly percent $80 \% \& 72 \%$ of patients in group $2 \& 1$ respectively.

Figure(1) : pulmonary function test in preoperative day (1st assessment) and fourth day ( $2^{\text {nd }}$ assessment) postoperatively, this figure mention that decreased FEV1\&VC in fourth day postoperatively than preoperative phase with a significant difference was found between the two groups during the first and second assessment related to $\mathrm{VC}$.

\section{Discussion}

Patient education is essential component of nursing practice, nurses play a vital role in the prevention of post-operative pulmonary complications in patient with open heart surgery.

Therefore, the ultimate goal of this study was examining the effect deep breathing maneuvers (incentive spirometer ,conservative therapy) on occurrence of postoperative pulmonary complications after open heart surgery.

Based on result of the present biosociodemograph characteristic, the mean age in group 1(incentive spirometer), 30-39 years old in group 2(concervative therapy) with no statistical significant difference between the two groups as regards to age. In 
relationship between age and pulmonary complications, the present study clarifies that pulmonary complications occur in age more than 50 years old because in this age patient have imuncompromasied and having chronic disease. (Urell et al., 2012) reported that younger patients had lower postoperative pulmonary complications after open heart surgery than older patients. This study on line with the present study.(Hulzebos et al., 2003) reported that preoperative risk factors for postoperative pulmonary complications( PPC )were an age of more than 70 years, This study on line with the present study.

The current study revealed that the percentage of male to female in the two groups the majority were male in group 2 with statically significant difference between two groups.

The present study clarifies that the majority of patient's diagnosis were RHD in group1, 2 with statically significant difference between two groups. (Tom et al, 2001) reported that no difference between the treatment group (incentive spirometer) and control group for either type of surgery,this study disagreement with the present study. In relationship between type of operations and pulmonary complications the current study revealed that pulmonary complication occurs in patients having CABG.

The finding of the current study revealed that there was no significant difference between two groups as regard smoking and there was no significant difference between two groups regarding body mass index (BMI).

The present study demonstrated that there was no significant difference between two groups in length of ICU stay. (Stiller \& Munday, 2008) found no significant difference in length of stay in hospital between treatment groups received regular breathing and coughing exercises, incentive spirometer. This study on line with present study, but (Possa et al, 2013) reported that the use of incentive spirometry decrease length of hospital stay compared control group. And the present study revealed that no significant difference as regard time of chest tube removal.

Chest physiotherapy \& hemodynamic parameter (vital signs)

Clinically, hemodynamic stability observed throughout the present study in the majority of the studied patients, parameters including heart rate, Systolic blood pressure, diastolic blood pressure, temperature and mean arterial pressure. Concerning the respiratory rate) statistical significant differences were found between the two groups during the first assessments .Regarding to body temperature, a statistical significant difference was found between the two groups during the first, third assessment.

(Stiller et al., 2010) reported that no significant differences between 3 groups using (IS, routine chest physiotherapy and PEP) as regard temperature at any stage of treatment during fourth postoperative day.

(leigh et al.,2006) said that Respiratory rate, was essentially equal in all three groups using (IS, routine chest physiotherapy and PEP).as regard temperature said that Those using the incentive spirometer maintained a higher temperature for a longer period. this result disagreement with my study.

Patient who had cardiothoracic surgery were intubated and received mechanical ventilation for extended periods often up to 24 hours or more .many institutions are currently extubated these patients earlier to prevent the adverse effects of prolonged intubation and reduce pulmonary complication, after that patient receive supplement oxygen via vent face mask or nasal cannula (lewis ,2006).

The finding of the present study clarifies that there was no significant difference between two groups as regard mechanical ventilator parameters (mode of MV, FIO2, PS,PEEP and frequency).

The current study emphasized that there was significant difference between two groups in assessment of respiratory system after extubation as regard presence of cough.

Pain after cardiac surgery can lead to poor inspiratory effort in spontaneously breathing patient, which may contribute to postoperative pulmonary dysfunction, optimal pain relief is essential to enable the patient to perform maximal inspiration (Morrow, 2010).

The present study clarifies that patients having mild pain while coughing with significant difference between 2 groups. (Moreno et al., 2011) reported that pain may contribute to decreased cough efficiency, which is the main mechanism for the elimination of secretion from the tracheobronchial tree due to the immobility of the thoracic wall, which result in atelectasis. This result agreement with the current study, But in another study (Westerdhl, 2004) reported that no significant difference between any of groups in $4^{\text {th }}$ postoperative day when Pain from the sternotomy as measured by VAS.

As regard laboratory investigation the present study revealed that there was significant difference between any of groups in WBC during $2^{\text {nd }}$ assessment (first day postoperative open heart surgery).

Effect of deep breathing maneuvers on gas exchange

ABG were measured immediately before extubation and before, after the deep breathing intervention on second postoperative day the patients showed mild hypoxia, but oxygenation improvement after preformed deep breathing intervention. The finding 
of the present study revealed that patient's oxygenation improved after preformed 2deep breathing maneuvers with significant difference between two groups in $1^{\text {st }}$ and $3^{\text {rd }}$ day postoperatively regarding $\mathrm{SaO}_{2}$, and significant difference between two groups in $4^{\text {th }}$ day regarding $\mathrm{paO}_{2}$.

(Hofmeryr et al., 2012) reported that there were no significant differences in arterial oxygen saturation between 3 groups, (Stiller et al., 2010) reported that $\mathrm{PaO}_{2}$ and $\mathrm{FIO}_{2}$ were significantly reduced on the first postoperative day. By fourth postoperative day oxygenation had improved. This study in line with the present study

(Leigh et al., 2006) reported that on the third postoperative day there was a significant improvement in $\mathrm{PaO} 2$ in the group using blow bottles and a lesser improvement in the groups using the incentive spirometer, this result disagreement with our study.

(Emtner et al., 2011) reported that arterial oxygen saturation $\left(\mathrm{SaO}_{2}\right)$ was increase in treatment group (deep breathing with incentive spirometer) than control group (deep breathing only).

(Westerdahi et al., 2004 ) reported that oxygenation had slightly improved by the fourth postoperative day with no statistical difference between 2 deep breathing maneuvers ( blow bottle and IS) and control group .

Effect of deep breathing maneuvers on pulmonary function test (PFT) and comparison between the 2 groups (blow bottle, IS\& conservative therapy)

Reduction in lung volumes and oxygenation are common during the initial period after open heart surgery. The effects of the median sternotomy, hypothermia for myocardial protection, dissection of internal mammary artery and the use of cardio pulmonary by pass negatively influence lung function (Banmgarten et al., 2009).

In comparison to preoperative value the current study showed that mean reduction observed in forced vital capacity (FVC), forced expiratory volume in 1 second (FEV1) \&vital capacity (VC) on fourth postoperative day compered to preoperative day. And the current study clarifies that a statically significant difference between two groups regarding to vital capacity (VC) with improved in(FVC ,FEV\& VC) in patients preforming deep breathing with IS than routine deep breathing and coughing exercise .

There are many studies supported this finding,(Jenkins et al., 2012)said that the pulmonary function after CABG were severely reduced in all treatment groups on the fourth postoperative day with a mean $60-75 \%$ of the preoperative values.

(Ragnarsdotti, 2004) showed that a 33\% decrease in pulmonary function on postoperative day 3 and a
$23 \%$ on postoperative day 6 compared to the preoperative period.(Moreno et al., 2011) reported that pulmonary function decreased after CABG, pulmonary function was the worst on postoperative day 3 and began to improve on postoperative day 15 .

(Weissman, 2004) reported that after cardiac surgery, there are decreases in forced vital capacity (FVC), expiratory volume in the first second of forced expiration $\left(\mathrm{FEV}_{1}\right)$ and maximum voluntary ventilation than preoperative period.

(Stockc et al., 2010) reported that patients who undergo upper abdominal and cardiac surgery operations experience proportional decreases in all lung volumes without clinically significant changes in $\mathrm{FEV}_{1}, \mathrm{FVC}$, but patients received IS had more rapid recovery of $\mathrm{VC}$ than those who received conservative therapy, but the two groups treatment groups showed similar improvements in FVC and FEV.(Nicholson et al., 2010) reported that a mean reduction on FVC \&FEV to be $40-50 \%$ on the first to third postoperative day, on the second day a mean reduction of $63 \%$ in vital capacity compered to preoperative day .

Chest physiotherapy

Effect of deep breathing maneuvers on pulmonary complications and comparison between the 2 groups (Incentive spirometerS \& conservative therapy)

The present study confirmed that the majority of complications are (pneumonia ,atalectsais, bronchitis ,fever and pulmonary secretions) respectively in group2(conservative therapy) with statistical significant difference between two groups in atelectasis and pulmonary secretions . Regarding pulmonary secretion it was noticed that a highly percent $80 \% \& 72 \%$ of patients in group $2 \& 1$ respectively.

(Wattie, 2010) repeated this survey and found that usage rate had increase to $71 \%$ despite need for IS in patients undergoing $\mathrm{CABG}$ surgery and the effectiveness of IS in decease pulmonary complication postoperatively. (Possa et al., 2013) reported that the use of incentive spirometry \& positive expiratory air way pressure decrease length of hospital stay compared control group.

(Hoeffener et al., 2008) two studies reported that the IS improved the lung function when compared to other physical therapy interventions, and one study showed that the IS did not restore the lung function after surgery when compared to the use of exercises with positive pressure (PEP).

(Thomas \& Mcintosh 2010) reported that incentive spirometery and deep breathing exercise appear to be more effective than no physical therapy intervention in the prevention of postoperative pulmonary complication. 
(Hoeffener et al., 2008) showed that the use of incentive spirometer reduced the incidence of PPC (postoperative pulmonary complication in patients undergoing surgery for myocardial revascularization when compared with deep breathing.

(Vilaplana et al., 2009) was observed that patient who used the IS associated with inspiratory muscle training in the postoperative period presented an improvement in lung function when compared to a control group without intervention.

(Yane - Brager et al., 2009) found no differences in the incidence of PPC when compared IS to another intervention. ( Celso et al., 2011) no evidence was found that support the use of IS in the management of surgical patients,this study disagree with present study.

In the fact that study documented that using of deep breathing exercise with incentive spirometer more effective for decreasing pulmonary complications for patients under open heart surgery.

\section{Conclusion}

Based on the results of the current study it can be concluded that a positive effects of incentive spirometer compared with other physiotherapy breathing techniques on occurrence of pulmonary complications among open heart patients postoperatively. Significant restrictive decrease in pulmonary function was present on the fourth postoperative day after CABG rather than preoperative day. Pain from the sternotomy was low and could not explain the impairment. Major differences were found between patients performing deep breathing exercise alone and deep breathing with incentive spirometer during the first four postoperative days. Regarding to secretions the study revealed that patients who performed deep breathing exercises with incentive spirometer postoperatively having small amount of secretions with statistical significant difference between the two groups.

\section{Recommendations}

Based on the finding of the current study, the following recommendations are suggested

- Provide training program to update critical care nurses knowledge and skill about new maneuvers of deep breathing.

- Critical care nurse must have the ability to make careful assessment for critical care patient after extubation about signs and symptoms of respiratory complications.

- Available critical care nurse especial for chest physiotherapy (physiotherapist) must be present in ICU.
- Repeat this research on a large sample size to evaluate effect of chest physiotherapy on preventing of respiratory complications after open heart surgery.

- Instructing the patient chest physiotherapy procedures preoperatively to allow understanding what should be done and why.

\section{References}

1. Bartlett R., Gazzaniga AB\&Geraghty T., (2010): Respiratory maneuvers to prevent postoperative pulmonary complications. A critical review. JAMA; 224(7):1017-1021.

2. Black J., \& Jacobs E., (2010): Medical surgical nursing clinical management for continuity of care $6^{\text {th }}$ ed. Philadelphia: Saunders company; 492 $-493$.

3. Celso R., Romoanhlo S., Manguerir B., Munhoz R., Manzano, \& Edson J., (2011): chest physiotherapy during immediate postoperative period among patients undergoing upper abdominal and thoracic surgery;126(5):269-273.

4. Curley M., Moloney I., \& Harmon P., (2006): critical care Nursing, $5^{\text {th }}$ ed., Philadelphia: Sanders company ; $208-212$.

5. Deborah leader R., (2008) : uses of incentive spirometer indication \& contraindication;89:652656.

6. Duggan M., \& Kavanagh B., (2010): Perioperative modifications of respiratory function. Best Pract Res ClinAnaesthesiol.; 24(2):145-55.

7. Emtner M., Vrell C., Hedenstrom H., tenling A., Breidenskog M., \&Westerdahl E., (2011): deep breathing with positive expiratory pressure rate improve oxygenation in the early period after cardiac surgery 40 (1): $162-7$.

8. Hery M., \& Thompson J., (2005): clinical nursing surgery; Edinburgh; Saunders: 252.

9. Hoeffener M., Ferreira G., Rarretoss, Arena B., \& Dall A., (2008) : incentive spirometer with expiratory air way pressure reduces pulmonary complication improves pulmonary function and 6- minute walk distances in patients undergoing coronary artery bypass graft Am Heart, 158 (6): $901-8$.

10. Hoeffener M., Ferreira G., Rarretoss, Arena B., \& DallAgop (2008) : incentive spirometer with expiratory air way pressure reduces pulmonary complication improves pulmonary function and 6- minute walk distances in patients undergoing coronary artery bypass graft Am Heart, 158 (6): $901-8$. 
11. Hofmeyr J., Webber B., \& Hodsonme (2012): evaluation of positive expiratory pressure as an adjunct to chest physiotherapy in the treatment of cystic fibrosis, 41: $951-954$.

12. Hulzebos E., Van Mceteren N., De Bie R., Dagnelie P., \&helders D., (2003): prediction of postoperative pulmonary complications on the basis of pre-operative risk factors in patients who had undergone coronary artery bypass graft surgery. 83(1); $8-16$.

13. Ingwersen U., Larsen K., Bertelsen M., KiilNielsen K., Laub M., Sandermann J., (2005) : Three different mask physiotherapy regimens for prevention of post-operative pulmonary complications after heart and pulmonary surgery. Intensive Care Med; 19:294-298.

14. Joehl R., (2005): Preoperative evaluation: pulmonary, cardiac, renal dysfunction and comorbidities. SurgClin North Am.; 85(6):106173.

15. Lawrence V., Hazuda H., Cornell J., Pederson T., Bradshaw P., Mulrow C., et al., (2004): Functional independence after major abdominal surgery in the elderly. J Am Coll Surg. 199(5):762-72.

16. Leigh I., Ieverson M., Roger R., Ecker N., Harold E., Fox M., \& Ivan A., (2006) : three different mask physiotherapy regimens for prevention of postoperative pulmonary complication after heart and pulmonary surgery. Intensive care Med: (25) 197 - 200.

17. Lewis C., (2006): Encyclopedia of respiratory medicine oxygen therapy: $278-82$.

18. Moreno A., Castro R., Sorares P., Anna M., Cravo S., \&Nobrega A., (2011) : Longitudinal evaluation the pulmonary function of the pre and postoperative periods in the coronary artery bypass graft surgery of patients treated with physiotherapy protocol $6: 62$.

19. Morrow L., (2010) : pulmonary complications after coronary bypass graft;51:215-218.

20. Nicholson J., Kowalski E., Hamilton G., Meyers P., Serrette C., and Duke P., (2010): postoperative pulmonary function in coronary artery bypass graft surgery patients undergoing early tracheal estuation. A comparison between short term mechanical ventilation and early extubation, journal of cardiothoracic and vascular anesthesia; 6 ( 1): $27-31$.

21. Pasquina P., \& Martin R., (2003): prophylactic respiratory physiotherapy after cardiac surgery ;327.

22. Possa S., Amador B., Gosta A., Sakamoto E., Kondos, Vasconcellos M., and Brito M., (2013) : using incentive spirometer and positive pressure after open heart surgery ;1( 29): 1 - 9 .
23. Possa S., Amador B., Gosta A., Sakamoto E., Kondos, Vasconcellos M., and Brito M., (2013) : vol. 29; 1 - 9 .

24. Ragnarsdotti M., Kristjansdottir A., Ingvarsdottil ,Hannesson P \&Torfason B., (2004) : Short tern changes in pulmonary function and respiratory movements after cardiac surgery via median sternotmy 38 (1): $46-52$.

25. Savci S., Sakinc S., inal D., Arikan H., Can Z., Buran Y., \&Kuralay E., (2006): active cycle of breathing techniques and incentive spirometer in coronary artery bypass graft surgery 17 (2) : 64 69.

26. SchullerD\&Marrow L., (2007) : pulmonary complications after coronary revascularization curropin, cardio ; 15 (5): 309 - 15.

27. Smetana G., (2009): Postoperative pulmonary complications, an update on risk assessment and reduction. Cleve Clin (76) 4: 60-65.

28. Stiller K., \&Munday R., (2008) : Chest physiotherapy for the surgical patient, (79): 745 $-749$.

29. Stiller K., Montarello J., Walace M., Daff M., Grant R., Jenkins S., Hall B., Yates H., \&Nurs B., (2010) : efficacy of breathing and coughing exercises in the prevention of pulmonary complications after coronary artery surgery 105 : $741-47$.

30. Stock C., John B., Downs M., Pauk, Joan M., Alster M., \& Peter B., (2010): prevention of postoperative pulmonary complications with CPAP, incentive spirometer and conservative therapy 87: $151-157$.

31. Thomas J., \& Mcintosh, (2010): incentive spirometry, intermihent positive pressure breathing land deep breathing exercises effective in the prevention of postoperative pulmonary complications after upper abdominal surgery 74 (1); 3 - 8

32. Thompson M., Mark A., \& Rasmus M., (2010): How to use incentive spirometery;204215.

33. Tom J., Overend, Catherine M., Anderson, Deborah Lucy L., Christina B\&TimmernanS, (2001), The effect of incentive spirometry on postoperative pulmonary complications; 120 (3): $971-978$.

34. Urell C., Hedentrom H., Westerdahl E., Janson C., \&Emtner M., (2012): lung function before and two days after open-heart surgery ; 23: $1-7$.

35. Vilaphana J., Sabale A., Ramon R., Gasolibe Z., \& Vilalooga B., (2009): infectiveness of incentive spirametry as coadjuvant of conventional physiotherapy for the prevention of postoperative respiratory complications after 
thoracic and esophageal surgery Rev. Fsp Anestosiol Reanim 37 (6): 321 - 5 .

36. Wattie J., (2010): incentive spirometery follow in coronary artery bypass surgery pp 84 .

37. Weindler J., \& Kiefer R., (2011): the efficacy of postoperative incentive spirometery is influenced by the device specific - imposed work of breathing chest 119 (6); $1858-1864$.

38. Weissman C., (2004) : pulmonary complications after surgery. 8(3); $185-211$.

39. Westerdahi L\&Orman J., (2010): chest physiotherapy with postoperative expiratory pressure breathing after abdominal and thoracic surgery; 54;461 - 467 .

40. Westerdahl E. Lindmark B, Almgren S., et al., (2004) : chest physiotherapy after coronary artery bypass graft surgery comparison of three different deep breathing techniques, J Rehabil med ; 33: $79-89$.

41. Westerdeh E\&Lindmark B., (2001): Chest physiotherapy after coronary artery bypasses graft surgery. A comparison of three different deep breathing;30:66-72.

42. Yane - Bragel, Pila - Fernandes, Juffe- Slein A ,Marhne. Gonzale U, Perlega Diaz S \& Mauleon Garcia A., (2009): Respiratory physiotherapy and incidence of pulmonary complications in off pump coronary artery bypass graft surgery an observational follow-up study. 